\title{
Editorial: L2 Phonology Meets L2 Pronunciation
}

\author{
John Archibald ${ }^{1 *}$, Mary Grantham O’Brien ${ }^{2}$ and Andrew Sewell ${ }^{3}$ \\ ${ }^{1}$ Department of Linguistics, University of Victoria, Victoria, BC, Canada, ${ }^{2}$ School of Languages, Linguistics, Literatures, and \\ Cultures, University of Calgary, Calgary, AB, Canada, ${ }^{3}$ Department of English, Lingnan University, Hong Kong SAR, China
}

Keywords: L2 phonology, L2 pronunciation, L2 speech, L2 perception and production, L2 pedagogy

\section{Editorial on the Research Topic}

\section{L2 Phonology Meets L2 Pronunciation}

The theme of this collection is "L2 Phonology Meets L2 Pronunciation." Such an interdisciplinary approach, of course, runs the risk of any gathering of friends at which you discover that your chessclub friends having nothing to say to your ultimate-frisbee teammates. We are sure, however, that these papers reveal this not to be the case here. In various guises as researchers and teachers, the three editors have tackled the question of what is easy and what is difficult in both learning and teaching (the sub-theme of the collection). We hope you find our introductory mini-reviews helpful in setting the stage. One is on pronunciation teaching (O'Brien), one on functional load (Sewell), and one on L2 phonology (Archibald). There are clear similarities in what L2 phonologists are interested in, and what $\mathrm{L} 2$ pronunciation teachers are interested in. All three of these themes are intertwined in the collection.

What we have assembled here are papers written by people who have been fascinated by these same questions. In these nine papers, there are some which focus on consonants (Cardoso et al.; Stefanich and Cabrelli; Zhang and Levis), some on vowels (Cebrian et al.; Munro), some on prosody (Ghosh and Levis; Liu and Reed), and some on teaching (Colantoni et al.; Kostromitina and Kang). We group them in this way to reflect the Commentaries by eminent scholars that appear after the papers. We thank Shea, Thomson, McGregor, and Sonsaat Hegelheimer for accepting our invitation to round out the Research Topic. Of course, many of the papers reveal that the boundary line between phonology and pronunciation is really quite blurry. Such is the reality of scholarly life.

Stefanich and Cabrelli look at the production of the Spanish alveopalatal nasal/n/by L1 English speakers. They illustrate the complex developmental path of acquisition of this new sound.

Zhang and Levis look at a less-studied consonantal pattern: the effects of a merger of $/ \mathrm{n} / \mathrm{and} / \mathrm{l} / \mathrm{in}$ the Southwestern Mandarin dialect of Chinese. They demonstrate that this L1 property affects production in Standard Mandarin differently than it affects English production.

Cardoso et al. look at the effects of different types of instruction on the acquisition of consonantal sequences. They show that the group which received instruction on the most marked structure fares the best.

Munro presents a fascinating data set of Cantonese learners of English tense/lax vowels, and shows that there is a great deal of individual and lexical variation which makes it very challenging to talk of a monolithic notion of difficulty.

Cebrian et al. probe the relationship between perceived similarity judgments of English tense/lax vowels by Spanish/Catalan native speakers and their perception and production. They discover that perceived similarity is not always a good predictor of discrimination ability.

Colantoni et al. draw on the pronunciation literature, and set out and illustrate some design principles for enhancing L2 Spanish intelligibility in the classroom. 
Kostromitina and Kang document pronunciation development in ESL immersion learners. They demonstrate the differential effects that immersion can have on learners of differing levels of proficiency, and discuss the curricular implications.

Ghosh and Levis examine the effects on intelligibility that different types of stress errors can have. The propose a Gravity Hierarchy to classify error types and suggest that errors affecting vowel quality should be prioritized in training.

Liu and Reed probe the complexity of the L2 intonational system via both production and processing measures. They explore possible bridges between L2 phonology and L2 pronunciation teaching.

As has been said many times before, there is no such thing as the L2 learner or the L2 classroom; learners vary, and classrooms vary. But just as in the broader fields of linguistics (i.e., across languages) and language acquisition (i.e., across learners), we seek to account for both global uniformity and local variation, we see that this is an issue in our collection as well. Some of the papers reveal commonalities in either the learner population (Stefanich and Cabrelli; Zhang and Levis) or the learner behavior (Ghosh and Levis) or the learning environment (Cardoso et al.; Colantoni et al.; Kostromitina and Kang). Other papers highlight the local variation within a given population (Cebrian et al.; Liu and Reed; Munro). We may find different profiles when we look at different levels of proficiency within a language class, or different similarity judgements within an L1 group. This clearly is an area of interest for both the L2 phonologist and the L2 pronunciation teacher or curriculum designer.

This is a collection that probes some difficult questions but will provide no easy answers. Nonetheless, we feel its insights are numerous for theory, research methodology, and classroom practice.
So, take down this e-book and explore it like you'd explore parallel sessions in a conference, or streets from a workshop venue you visited years ago. You may come across some old friends, you will make some new and exciting discoveries, you might make a note to come back to certain things later, and you could find yourself at times nodding, at times questioning, this is as it should be. L2 phonology meet L2 pronunciation.

\section{AUTHOR CONTRIBUTIONS}

All authors listed have made a substantial, direct, and intellectual contribution to the work and approved it for publication.

\section{ACKNOWLEDGMENTS}

We would like to thank all the authors, reviewers, and editors who worked so diligently to help us bring this collection together.

Conflict of Interest: The authors declare that the research was conducted in the absence of any commercial or financial relationships that could be construed as a potential conflict of interest.

Publisher's Note: All claims expressed in this article are solely those of the authors and do not necessarily represent those of their affiliated organizations, or those of the publisher, the editors and the reviewers. Any product that may be evaluated in this article, or claim that may be made by its manufacturer, is not guaranteed or endorsed by the publisher.

Copyright (C) 2021 Archibald, O'Brien and Sewell. This is an open-access article distributed under the terms of the Creative Commons Attribution License (CC BY). The use, distribution or reproduction in other forums is permitted, provided the original author(s) and the copyright owner(s) are credited and that the original publication in this journal is cited, in accordance with accepted academic practice. No use, distribution or reproduction is permitted which does not comply with these terms. 